\title{
Safety and efficacy of interferon-ribavirin combination therapy in HCV-HIV coinfected subjects: an early report
}

H Zylberberg, Y Benhamou, J L Lagneaux, A Landau, M-L Chaix, H Fontaine, M Bochet, T Poynard, C Katlama, G Pialoux, C Bréchot, S Pol

\begin{abstract}
Background-More severe liver disease together with a poor response rate to $\alpha$ interferon argue for the use of more potent anti-hepatitis $\mathrm{C}$ virus $(\mathrm{HCV})$ therapies in human immunodeficiency virus (HIV)-HCV coinfected patients, but the efficacy and safety of interferonribavirin combination therapy in HIV infected subjects are unknown.

Aim-To retrospectively evaluate the efficacy and safety of anti-HCV combination therapy in 21 HCV-HIV coinfected patients receiving antiretroviral therapy, and to access the clinical relevance of in vitro inhibition of phosphorylation by ribavirin of potent inhibitors of HIV-that is, zidovudine, stavudine, and zalcitabine. Patients-Twenty one patients were treated with combined antiretroviral therapy including zidovudine $(n=8)$ or stavudine $(n=13)$ (in association with protease inhibitors in 12). All received ribavirin (1000 or $1200 \mathrm{mg} / \mathrm{day}$ ) and $\alpha$ interferon (3 MU three times/week) for chronic hepatitis $C$ infection. All patients had not responded $(n=20)$ or relapsed $(n=1)$ after a previous six month course of $\alpha$ interferon therapy.
\end{abstract}

Methods-HIV viral load (Monitor test) and CD4 cells count were measured at the beginning and every three months during and after ribavirin plus $\alpha$ interferon therapy over a mean period of 11 (1) months. Clinical and biological adverse effects were recorded.

Results-There was no significant variation in HIV viral load or CD4 cell counts after three or six months of ribavirin therapy compared with baseline values. Of the 21 subjects, three (14\%) had an increase in HIV viral load of more than 0.5 log leading to discontinuation of ribavirin in one. Eleven of $21(52.4 \%)$ had initial negative HCV viraemia at three $(n=10)$ or six $(n=1)$ months but only six were polymerase chain reaction negative at the end of therapy, leading to rates for primary response and breakthrough of $23.8 \%$ and $28.5 \%$, respectively. Six months after completion of therapy, three patients relapsed $(14.3 \%)$ and three $(14.3 \%)$ had sustained virological response. Median haemoglobin concentration decreased significantly after three and six months of ribavirin therapy $(p=0.0002$ and $p=0.0003$, respectively) leading to withdrawal of therapy in one patient.

Conclusions-These preliminary results show that: (1) despite in vitro interactions between ribavirin, zidovudine, and stavudine, significant variation in HIV replication does not usually occur in HCV-HIV coinfected patients receiving ribavirin and different antiretroviral regimens, including zidovudine and stavudine; (2) $\alpha$ interferon and ribavirin combination therapy induced primary and sustained virological responses in $28.5 \%$ and $14.3 \%$ of treated subjects (who were previous non-responders to interferon therapy), respectively; (3) anaemia is a frequent adverse event. Such results should be confirmed in larger prospective trials.

(Gut 2000;47:694-697)

Keyword: ribavirin; human immunodeficiency virus; hepatitis C virus; zidovudine; stavudine; antiretroviral therapy

Hepatitis C (HCV) and human immunodeficiency (HIV) viruses share the same routes of transmission, explaining the high rate of $\mathrm{HCV}$ and HIV coinfection of $9-30 \% .{ }^{1} \mathrm{HCV}$ related disease is more severe in HIV than in non-HIV infected individuals with a twofold higher rate of cirrhosis, ${ }^{2}$ and may be associated with an increased risk of liver related death. ${ }^{3}$

More severe liver disease together with a poor response rate to $\alpha$ interferon of HIV-HCV coinfected patients ${ }^{1}$ argue for the use of more potent anti-HCV therapies to avoid histopathological deterioration. Anti-HCV combination therapy with interferon and ribavirin has shown promising results in immunocompetent (naive and relapser) patients with a marked increase in the virological sustained response rate ${ }^{4-7}$ but the efficacy and safety of such combinations in coinfected subjects are unknown.

In vitro inhibition of phosphorylation by ribavirin of HIV reverse transcriptase inhibitor (that is, zidovudine (AZT), stavudine (D4T), and zalcitabine) to the active compound has been reported..$^{8-10}$ The clinical impact of these findings is currently unknown and it may be hypothesised that ribavirin impairs the efficacy of antiretroviral drugs. Moreover, there may be

Abbreviations used in this paper: HIV, human immunodeficiency virus; $\mathrm{HCV}$, hepatitis $\mathrm{C}$ virus; $\mathrm{AZT}$, zidovudine; D4T, stavudine; PCR, polymerase chain reaction. 
Table 1 Baseline characteristics of human immunodeficiency virus-hepatitis $C$ virus (HIV-HCV) coinfected patients

\begin{tabular}{lc}
\hline Mean (SD) age (y) & $40.2(7)$ \\
Male & $17(81 \%)$ \\
Risk factor for HIV-HCV infections & $17(81 \%)$ \\
$\quad$ Intravenous drug use & $3(14.3 \%)$ \\
$\quad$ Transfusion & $8(38 \%)$ \\
Antiretroviral therapy & $13(62 \%)$ \\
$\quad$ Zidovudine & $12(57 \%)$ \\
$\quad$ Stavudine & \\
HIV-1 protease inhibitor & $8.3(6.0-10.5)$ \\
Duration of antiretroviral therapy (months) & $3-22$ \\
$\quad$ Median $(95 \%$ CI) & $11(52.4 \%)$ \\
$\quad$ Range & \\
Cirrhosis (No (\%)) & \\
\hline
\end{tabular}

a greater risk of anaemia in view of the haemolysis associated with ribavirin and the bone marrow suppression associated with nucleoside analogues. The aims of this study were: (1) to retrospectively evaluate the safety of anti-HCV combination therapy, especially the clinical relevance of these in vitro interactions, by reviewing the clinical, biological, virological, and immunological outcome of 21 coinfected patients receiving an antiretroviral regimen, including at least D4T or AZT; and (2) to evaluate the efficacy of interferon and ribavirin combination therapy on $\mathrm{HCV}$ replication.

\section{Patients and methods}

PATIENTS

Twenty one HCV-HIV coinfected patients receiving antiretroviral therapies, including at least D4T $(n=8)$ or AZT $(n=13)$, for a median period of 8.3 (3-22) months prior to introduction of ribavirin plus alpha interferon were studied. The main characteristics of the patients are summarised in table 1 . There were 17 men and four women with a mean age of 40 (7) years. Risk factors for viral transmission were intravenous drug usage in 17 , transfusion in three, and sexual contact in one. HCV genotype was $3 a(n=7), 1 a(n=6), 1 b \quad(n=4), 4$ $(n=2)$, and $2(n=2)$. Twelve $(57 \%)$ patients were treated with a HIV-1 protease inhibitor, including indinavir $(n=5)$, saquinavir $(n=5)$, or ritonavir $(n=4)$ (two were given both ritonavir and saquinavir), in association with two nucleoside analogues.

Liver biopsy showed cirrhosis in 12 of 21 patients $(57 \%)$ and mean activity and fibrosis scores were 2 (1) and 3 (1), respectively.

All patients had not responded $(n=20)$ or relapsed $(n=1)$ after a previous six month course of $\alpha$ interferon therapy. Patients re- ceived anti-HCV therapy for a mean period of 8.5 (3) months, including ribavirin (1000 or $1200 \mathrm{mg}$ daily) and $\alpha$ interferon (3 MU subcutaneously three times weekly).

MEASUREMENTS

HIV RNA plasma levels were assessed with a standard available commercial assay (Amplicor HIV-1 Monitor, Roche Diagnostic System Neuilly sur Seine, France) which has a detection limit of 200 copies of HIV-1 $\mathrm{RNA} / \mathrm{ml}$. HCV polymerase chain reaction (PCR) was performed using a standard commercial assay (Amplicor HCV Monitor, Roche Diagnostic System Neuilly sur Seine, France). CD4 lymphocyte counts were measured using dual colour flow cytometry.

HIV viral load and CD4 cell counts were measured three months before, at the beginning of the study, at three month intervals after ribavirin plus $\alpha$ interferon therapy, and three months after completion of therapy over a mean period of 9 (1) months.

STATISTICAL ANALYSIS

Changes in median HIV viral loads, CD4 counts, and haemoglobinaemia were compared by the Wilcoxon signed rank test. Results are expressed as median (range).

\section{Results}

Median HIV load and CD4 counts, performed in 21 patients three months before starting interferon and ribavirin therapy, were 2.8 (2.35.9) $\log 10$ copies/ml and 293 (45-655) cells/ $\mathrm{ml}$, respectively. At the beginning of ribavirin and interferon therapy, CD4 counts were not different and HIV RNA plasma levels were significantly lower than three months earlier $(\mathrm{p}=0.3$ and $\mathrm{p}=0.05$, respectively).

There was no statistically significant change in HIV viral load or CD4 cell counts after three or six months of ribavirin therapy compared with baseline values (table 2, fig 1). However, an increase of more than $0.5 \mathrm{log}$ copies $/ \mathrm{ml}$ of plasma HIV-RNA (1.3, 0.6, and 0.6, respectively) was observed in three patients (14\%) after 3-6 months of ribavirin. Two of these three patients had undetectable HIV viraemia prior to ribavirin. After three months of ribavirin plus interferon therapy, a median decrease of 68 (23-97) CD4 lymphocytes/ml was observed in 11 of 21 patients $(52.4 \% ; 95 \%$ CI $29.7-74.3 \%)$. After six months of anti-HCV

Table 2 Biological, virological, and immunological outcomes of human immunodeficiency virus-hepatitis $C$ virus $(H I V-H C V)$ coinfected patients receiving interferon and ribavirin therapy

\begin{tabular}{|c|c|c|c|c|c|}
\hline & Baseline & Month 3 & $p$ Value ${ }^{\star}$ & Month 6 & $p$ Value ${ }^{\star}$ \\
\hline $\mathrm{n} \dagger$ & 21 & 21 & & 17 & \\
\hline \multicolumn{6}{|l|}{ Haemoglobinaemia $(\mathrm{g} / \mathrm{dl})$} \\
\hline Median (range) & $13.8(10.5-16.7)$ & $11.8(7.8-16)$ & 0.0002 & $11.8(5-16)$ & 0.0003 \\
\hline $\mathrm{n} \dagger$ & 21 & 21 & & 17 & \\
\hline RNA HIV (log10 copies/ml) & $2.3(2.3-4.9)$ & $2.3(2.3-4.6)$ & 0.3 & $2.3(2.3-4.7)$ & 0.6 \\
\hline HIV RNA negative (n (\%)) & $12(57)$ & $10(47.6)$ & & $10(47.6)$ & \\
\hline \multicolumn{6}{|l|}{ CD4 $($ cells $/ \mathrm{ml})$} \\
\hline $\mathrm{n} \dagger$ & 21 & 21 & & 17 & \\
\hline Median (range) & $330(55-600)$ & $336(32-639)$ & 0.6 & $298(55-507)$ & 0.2 \\
\hline \multicolumn{6}{|l|}{ HCV PCR } \\
\hline $\mathrm{n} \dagger$ & 21 & 21 & & 17 & \\
\hline No patients with negative PCR (\%) & $0(0)$ & $10(47)$ & & $6(35)$ & \\
\hline
\end{tabular}

${ }^{\star}$ Compared with baseline.

†Number of tested patients. 


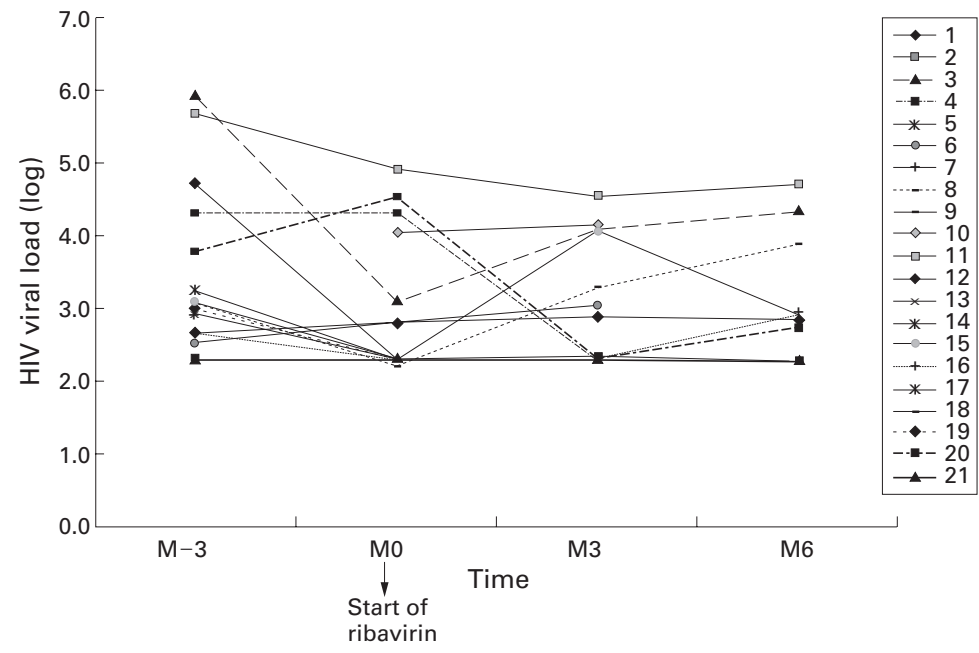

Figure 1 Human immunodeficiency virus (HIV) viral load three months before, at the start of ribavirin therapy, and three and six months after ribavirin therapy in individual patients $(n=21)$. that ribavirin inhibits intracellular phosphorylation of AZT, D4T, and zalcitabine, suggesting a decrease in their anti-HIV efficacy. ${ }^{8-10}$ The underlying molecular basis of such inhibition is under investigation and probably involves inhibition of thymidine kinase activity. ${ }^{10}$

The association of ribavirin and interferon with AZT or D4T for 3-6 months did not induce a significant change in HIV viral load or CD4 lymphocyte counts. This finding suggests that interactions between ribavirin and AZT or D4T may not influence HIV replication in vivo. However, three patients had an increase in HIV load of more than $0.5 \mathrm{log}$ which could be related to impairment of AZT or D4T efficacy due to inhibition of phosphorylation and which led to discontinuation of ribavirin in one patient. It is noteworthy that an increase in HIV-1 RNA plasma levels cannot be related definitively to ribavirin but may be related to HIV resistance to antiretroviral therapy or to poor compliance with therapy. Similarly, as previously reported, the decrease in CD 4 cell counts observed in $50 \%$ of patients was probably related to $\alpha$ interferon ${ }^{1}$ rather than to ribavirin. Indeed, a decrease in CD4 cell counts was not observed in large trials in which HIV infected patients received only ribavirin. ${ }^{1112}$

Analysis of efficacy of anti-HCV combination therapy in coinfected subjects showed $23.8 \%$ and $14.3 \%$ primary (HCV-RNA clearance at the end of therapy) and sustained virological response rates, respectively, in patients who were previous non-responders to interferon therapy. This very low sustained rate of response in non-responders parallels those obtained in the general population. ${ }^{13}$ Eleven of $21(52 \%)$ patients had initial negative HCV viraemia during therapy. We believe that the discrepancies between an anti-HCV antiviral effect, at least initially, and the fact that many of these patients were interferon relapsers or nonresponders could be explained, at least in part, by better immune status at the time of the combination therapy than at the start of interferon monotherapy (data not shown). Indeed, it is noteworthy that most patients $(80 \%)$ received interferon therapy before HAART was available; most patients had a poorer immune status at the time of the first anti-HCV therapy. Moreover, among patients with initial negative HCV viraemia, nearly half experienced a breakthrough. Interferon and ribavirin therapy was well tolerated, except for ribavirin related haemolysis which requires monitoring of haemoglobin concentration and perhaps a decrease in initial dose to $600-800 \mathrm{mg} / 24$ hours.

In conclusion, our preliminary data suggest that short term treatment with ribavirin is feasible with no major clinical impact on HIV disease in patients receiving zidovudine and zalcitabine but requires close monitoring of HIV viral load. Therapeutic trials with longer follow up and in vitro evaluation are warranted to confirm the safety and efficacy of anti-HCV combination therapy in coinfected subjects. 
1 Zylberberg H, Pol S. Reciprocal interactions between human immunodeficiency virus and hepatitis $C$ virus infections. Clin Infect Dis 1996;23:1117-25.

2 Pol S, Lamorthe B, Trinh Thi N, et al. Retrospective analysis of the impact of HIV infection and alcohol use on chronic hepatitis $\mathrm{C}$ in a large cohort of drug users. $f$ Hepatol 1998;28:945-50.

3 Darby SC, Ewart DW, Giangrande PLF, et al. Mortality from liver cancer and liver disease in haemophilic men and boys in UK given blood products contaminated with hepatitis C. Lancet 1997;350:1425-31.

4 Reichard O, Gunnar N, Fryden A, et al. Randomised, double-blind, placebo-controlled trial of interferon $\alpha-2 b$ with and without ribavirin for chronic hepatitis C. Lance 1998;351:83-7.

5 Poynard T, Marcellin P, Lee SS, et al. Randomised trial of interferon $\alpha 2 \mathrm{~b}$ plus ribavirin for 48 weeks or for 24 weeks versus interferon $\alpha 2 \mathrm{~b}$ plus placebo for 48 weeks for treatment of chronic infection with hepatitis $\mathrm{C}$ virus. Lancet 1998;352:1426-32.

6 Mc Hutchinson JG, Gordon SC, Schiff ER, et al. Interferon alfa- $2 b$ alone or in combination with ribavirin as initial treatment for chronic hepatitis C. N Engl F Med 1998;339: treatmen

7 Davis GL, Esteban-Mur R, Rustgi V, et al. Interferon alfa-2b alone or in combination with ribavirin for the treatment of relapse of chronic hepatitis C. $N$ Engl 7 Med 1998;339: 1493-9.

8 Vogt MW, Hartson KL, Furman PA, et al. Ribavirin antagonizes the effect of azidothymidine on HIV replication. Science 1987,235:1376-9.

9 Baba M, Pauwels R, Balzarini J, et al. Ribavirin antagonizes inhibitory effects of pyrimidine 2'3'-dideoxynucleosides but enhances inhibitory effects of purine 2',3'dideoxynucleosides on replication of human immunodeficiency virus in vitro. Antimicrob Agents Chemother 1987; 31:1613-18

10 Hoggard PG, Kewn S, Barry MG, et al. Effects of drugs on 2',3'-didehydrothymidine phosphorylation in vitro. Antimicrob Agents Chemother 1997;41:1231-6.

11 Spanish Ribavirin Trial Group. Comparison of ribavirin and placebo in CDC group III human immunodeficiency virus infection. Lancet 1991;338:6-9.

12 Roberts RB, Hollinger FB, Parks WP, et al. A multicenter clinical trial of oral ribavirin in HIV-infected people with lymphadenopathy: virologic observations. AIDS 1990;4: lymphader.

13 Pol, S, Couzigou, P, Bourliere M, and a multicenter study group under the coordination of the Necker Hospital. A randomized trial of ribavirin and interferon- $\alpha$ vs. interferon- $\alpha$ alone in patients with chronic hepatitis $C$ who were non-responders to a previous treatment. 7 Hepatol 1999;31:1-7. 